\title{
Z-dependent crossing of excited-state energy levels in highly charged galliumlike lanthanide atomic ions
}

\author{
Fumihiro Koike* \\ Department of Materials and Life Sciences, Sophia University, 7-1 Kioi-cho, Chiyoda-ku, Tokyo 102-8554, Japan \\ Chihiro Suzuki $\odot{ }^{\dagger}$ Izumi Murakami $\odot,^{\dagger}$ Daiji Kato $\odot,^{\S}$ and Naoki Tamura $\odot^{\dagger}$ \\ National Institute for Fusion Science, National Institutes of Natural Sciences, 322-6 Oroshi-cho, Toki, Gifu 509-5292, Japan \\ Nobuyuki Nakamura \\ Institute for Laser Science, The University of Electro-Communications, 1-5-1 Chofugaoka, Chofu, Tokyo 182-8585, Japan
}

(Received 16 September 2021; revised 22 November 2021; accepted 19 January 2022; published 4 March 2022)

\begin{abstract}
Extreme ultraviolet (EUV) optical transitions of lanthanide highly charged ions have been studied. The $[\mathrm{Ni}] 4 s^{2} 4 p-[\mathrm{Ni}] 4 s^{2} 4 d$ and $[\mathrm{Ni}] 4 s^{2} 4 p-[\mathrm{Ni}] 4 s 4 p^{2}$ transition lines of galliumlike lanthanide elements have been spectroscopically measured in large helical device (LHD) plasma and electron-beam ion trap (EBIT) plasma. The wavelengths for Eu ions agreed within $0.03 \%$ between the LHD and EBIT measurements. Due to the enhancement of spin-orbit interactions along with the increase of atomic number $Z$ in $4 p$ atomic orbitals, energy splitting between $4 p_{1 / 2}$ and $4 p_{3 / 2}$ orbitals increases significantly from lanthanum to lutetium, causing the crossing of $Z$-dependent wavelength curves. The spectral line positions and strengths are theoretically calculated by means of the multiconfiguration Dirac-Fock method. The mixing of the [Ni] $4 s^{2} 4 d$ and [Ni] $4 s 4 p^{2}$ configurations leads to an avoided crossing in the apparent wavelength curves. The configuration mixing makes both the $[\mathrm{Ni}] 4 s^{2} 4 p-[\mathrm{Ni}] 4 s^{2} 4 d$ and $[\mathrm{Ni}] 4 s^{2} 4 p-[\mathrm{Ni}] 4 s 4 p^{2}$ lines well visible in the EUV spectra near the level crossing point. The levels are found to cross between $Z=62$ and 63 .
\end{abstract}

DOI: 10.1103/PhysRevA.105.032802

\section{INTRODUCTION}

The spectral data and the knowledge of dynamics in extreme ultraviolet (EUV) optical emissions from highly charged heavy ions are of interest in a variety of fields such as the development of short-wavelength light sources for semiconductor lithography [1], the development of microscopes using shorter-wavelength light sources [2], or the diagnostics of nuclear fusion plasma [3,4] in magnetic confinement, as well as inertial confinement reactors. Among the highly charged atomic ions of heavier elements, lanthanide atomic ions are considered as potential EUV light source elements for a shorter-wavelength range. They have been a subject of extensive spectroscopic study for a couple of decades [5-8].

\footnotetext{
*koikef@sophia.ac.jp

Also at Department of Fusion Science, The Graduate University for Advanced Studies, SOKENDAI, 322-6 Oroshi-cho, Toki, Gifu 509-5292, Japan.

${ }^{\ddagger}$ Also at Interdisciplinary Graduate School of Engineering Sciences, Kyushu University, Kasuga, Fukuoka 816-8580, Japan.

${ }^{\S}$ Also at National Institute for Fusion Science, National Institutes of Natural Sciences, 322-6 Oroshi-cho, Toki, Gifu 509-5292, Japan.

Published by the American Physical Society under the terms of the Creative Commons Attribution 4.0 International license. Further distribution of this work must maintain attribution to the author(s) and the published article's title, journal citation, and DOI.
}

In the region of $4 d$ or $4 f$ open valence subshell ionic states of lanthanide elements, narrow unresolved transition array (UTA) spectra from the complex of $4 d-4 f$ and $4 p-4 d$ optical transitions are observed [9-12], which may match the wavelength range of the maximum reflectivity in currently available multilayer mirrors for semiconductor lithography. Due to strong correlations among the electrons in open $N$ shells, in other words, strong configuration interactions between the different electron configurations in $N$-open-shell atomic ions [13], the center of the UTA spectra suffers a significant shift in energy from the value that is expected in the simplest atomic structure calculations [14].

For higher charged ions such as $4 s$ or $4 p$ open valence subshell ionic states of lanthanide elements, isolated lines are mainly observed $[3,4]$. In those ions, the relativistic effects in atomic orbitals come to play an important role. Specifically, the spin-orbit splittings between $4 p_{\frac{1}{2}}$ and $4 p_{\frac{3}{2}}$ atomic orbitals become significant for higher atomic numbers in lanthanide elements. Because the $p$-orbital wave functions extend into the region close to the atomic nucleus, they suffer a strong relativistic effect, if $Z$ is large. The valence excited electronic states may have different orders in energy for different atomic numbers; we may observe the energy-level crossings along with the change of atomic number $Z$. Zilitis [15] has discussed the $Z$-dependent energy-level crossings for a wide range of Ga-like isoelectronic series of atomic ions, although for $L$ shells Kato et al. [16] have discussed the Z-dependent energy-level crossings in highly charged neonlike heavy ions. They pointed out the importance of spin-orbit interactions in 
$2 p$ orbitals. Nakamura et al. [17] have discussed the configuration mixing at the level crossing in highly charged neonlike ions.

Extensive studies of EUV emissions from lanthanide ions have been performed in laser produced plasmas [5,9,11,18$21]$, in vacuum spark discharges $[5,10]$, and in tokamak plasmas [11,20-22]. Recently, systematic studies of EUV spectra from highly charged heavy ions have been carried out in large helical device (LHD) plasmas, a magnetically confined torus device of National Institute for Fusion Science (NIFS) by our group [3,4,23-30]. Further, charge-separated spectroscopy measurements for lanthanide ions have recently been carried out in electron-beam ion traps (EBITs) [31-36].

In the present paper, we consider several EUV emission lines which are realized by transitions of lower excited states of Ga-like lanthanide atomic ions to their ground states. The valence excited $\left[[\mathrm{Ni}] 4 s^{2} 4 d\right]_{\frac{3}{2}}$ electronic configuration undergoes strong configuration interaction with the subvalence shell excited electronic configuration $\left[[\mathrm{Ni}] 4 s 4 p_{+}^{2}\right]_{\frac{3}{2}}$ when their expectation energies are close to each other. Here, [Ni] represents the configuration $1 s^{2} 2 s^{2} 2 p^{6} 3 s^{2} 3 p^{6} 3 d^{10}$, and we use the relativistic notations for atomic orbitals; $4 d_{-}$and $4 p_{+}$stand for the $4 d$ spin-down and the $4 p$ spin-up relativistic orbitals, respectively. We use the same convention also for other atomic orbitals in the present paper. In this way, we can clearly distinguish the $\left[[\mathrm{Ni}] 4 s 4 p_{+}^{2}\right]_{\frac{3}{2}}$ state from the $\left[[\mathrm{Ni}] 4 s 4 p_{-} 4 p_{+}\right]_{\frac{3}{2}}$ state, in which the former is "optically forbidden" whereas the latter is "optically allowed" for transitions into the ground $\left[[\mathrm{Ni}] 4 s^{2} 4 p_{-}\right]_{\frac{1}{2}}$ state. The configurations $\left[[\mathrm{Ni}] 4 s^{2} 4 d_{-}\right]_{\frac{3}{2}}$ and $\left[[\mathrm{Ni}] 4 s 4 p_{+}^{2}\right]_{\frac{3}{2}}$ are mixed and provide us with two atomic states that consist of their linear combinations. Because the $Z$ dependences of the two configuration states are different, their energy curves may cross at a certain $Z$. Near the crossing point, the energy levels may repel each other due to the configuration interaction, leading to an avoided crossing in $Z$-dependent energy curves. We may observe an anomaly in the $Z$ dependence of the wavelengths for transitions of those atomic states. Furthermore, the configuration mixing of the excited-state configurations makes the optically forbidden $\left[[\mathrm{Ni}] 4 s^{2} 4 p_{-}\right]_{\frac{1}{2}}-\left[[\mathrm{Ni}] 4 s 4 p_{+}^{2}\right]_{\frac{3}{2}}$ transition visible due to the contribution of the optically allowed $\left[[\mathrm{Ni}] 4 s^{2} 4 p_{-}\right]_{\frac{1}{2}}-\left[\left[\mathrm{Ni} 4 s^{2} 4 d_{-}\right]_{\frac{3}{2}}\right.$ transition, because the actual excited atomic state is a linear combination of $\left[[\mathrm{Ni}] 4 s 4 p_{+}^{2}\right]_{\frac{3}{2}}$ and $\left[[\mathrm{Ni}] 4 s^{2} 4 d_{-}\right]_{\frac{3}{2}}$ configurations. This feature of the spectral lines produces complexities and difficulties in the analysis of the experimental spectra.

In the present paper, we conducted two kinds of complementary experiments. We measured the spectral lines of Ga-like lanthanide atomic ions in the LHD plasmas at a relatively higher-temperature range $0.9-2.4 \mathrm{keV}$. We also measured the Ga-like spectral lines of Eu ions in the EBIT plasmas. We performed a series of theoretical calculations based on the multiconfiguration Dirac-Fock (MCDF) method. We describe the experimental procedure and the results in Sec. II. We describe the theory and the method of numerical procedures in Sec. III. In Sec. IV, we describe and discuss the representative results of the present experiment and theoretical calculation. Finally, in Sec. V we give concluding remarks.

\section{EXPERIMENT}

\section{A. LHD experiment}

One of the spectral measurements has been carried out in the LHD plasma, taking advantage of its brightness and stability as a light source. The experimental procedure is basically the same as those described in our previous papers $[3,4,23-30,37,38]$. In LHD, large-volume $\left(\simeq 30 \mathrm{~m}^{3}\right)$ hydrogen plasmas of a torus shape are produced mainly by neutral beam injection (NBI) heating in the magnetic field of about $2.75 \mathrm{~T}$, generated by superconducting coils $[39,40]$. The total heating power and duration of the NBI are typically 10-13 MW and 2-4 s, respectively. Small amounts ( $\simeq 10^{17}$ atoms $)$ of the lanthanide elements are introduced into the plasmas by using a tracer-encapsulated solid pellet $[41,42]$. Electron temperature and density profiles are precisely measured by a high-resolution Thomson scattering diagnostic system $[43,44]$. The typical electron temperature and density of the core plasmas investigated in this paper are $1-2 \mathrm{keV}$ and $4 \times 10^{19}-6 \times 10^{19} \mathrm{~m}^{-3}$, respectively. Therefore, the concentration of the impurity ions is less than $0.1 \%$, assuming that the impurities are uniformly expanded in the plasma. It also means that the LHD plasmas are optically thin for the spectral lines emitted from the impurities.

The EUV spectra were measured by a 2-m SchwobFraenkel grazing incidence spectrometer called SOXMOS [45]. The SOXMOS is equipped with a $600-\ell / \mathrm{mm}$ grating and a microchannel plate (MCP) detector, the opposite side of which is coated with a phosphor screen. The spectral image appearing on the phosphor screen is transmitted to a linear photodiode array via a bundled optical fiber conduit. A desired spectral band (with a 3-4-nm bandwidth) is selectable by moving the MCP along a Rowland circle. The time evolution of the spectra of a selected band was recorded sequentially every 0.1 or $0.2 \mathrm{~s}$. The wavelength resolution of the SOXMOS is approximately $0.01 \mathrm{~nm}$, which is mainly determined by the instrumental width, due to the spread of electron clouds inside the MCP. The absolute wavelength calibration was performed using the positions of several reference lines of various impurities (carbon, nitrogen, neon, iron, etc.). A third-order polynomial fitting of these reference lines gave the wavelength uncertainty of about $0.005 \mathrm{~nm}$.

We measured the EUV spectra for almost all the lanthanide elements in our series LHD experiments [3,4,23-30] including the present paper. The typical EUV spectra of $\operatorname{Ce}(Z=58), \operatorname{Nd}(Z=60), \operatorname{Eu}(Z=63), \operatorname{Tb}(Z=65)$, and $\operatorname{Ho}(Z=67)$ ions in a wavelength range $6-10 \mathrm{~nm}$ are shown in Fig. 1. Because the spectra in Fig. 1 are observed in relatively high-temperature conditions, they are composed of discrete lines, due mainly to $\mathrm{Cu}-, \mathrm{Zn}-$, and Ga-like ions. The identification results of all the lines of $\mathrm{Cu}-, \mathrm{Zn}-$, and $\mathrm{Ga}-$ like ions will be reported in one of our forthcoming papers. In the present paper, we will focus on the two lines from the Ga-like ions for the $\left[[\mathrm{Ni}] 4 s^{2} 4 p_{-}\right]_{\frac{1}{2}}-\left[[\mathrm{Ni}] 4 s^{2} 4 d_{-}\right]_{\frac{3}{2}}$ and $\left[[\mathrm{Ni}] 4 s^{2} 4 p_{-}\right]_{\frac{1}{2}}-\left[[\mathrm{Ni}] 4 s 4 p_{+}^{2}\right]_{\frac{3}{2}}$ transitions. The lines identified as the former transition are indicated by arrows in Fig. 1. As for the latter transition, we have identified lines for $\mathrm{Ce}(Z=$ $58), \operatorname{Pr}(Z=59), \operatorname{Nd}(Z=60), \operatorname{Sm}(Z=62)$, and $\operatorname{Eu}(Z=63)$. A slightly broadened line at $7.367 \mathrm{~nm}$ for Eu ions in Fig. 1 is 


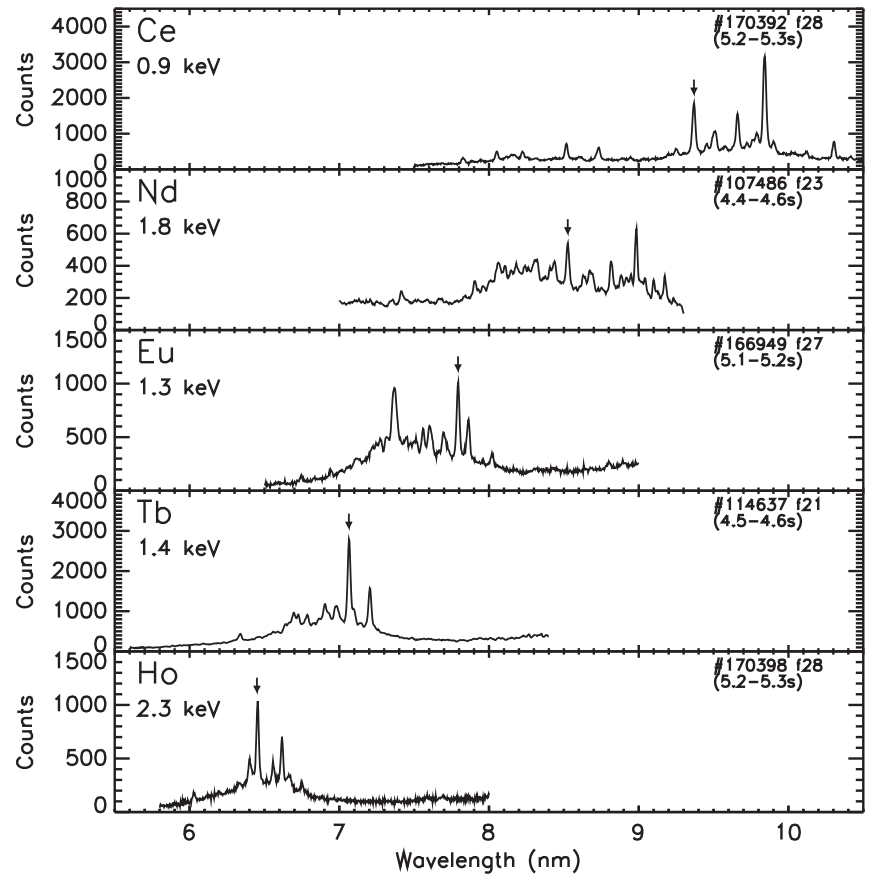

FIG. 1. Discrete EUV emission spectra of highly charged Ce, $\mathrm{Nd}, \mathrm{Eu}, \mathrm{Tb}$, and $\mathrm{Ho}$ ions in LHD. The vertical arrows indicate the lines due to the transition $\left.[\mathrm{Ni}] 4 s^{2} 4 p_{-}\right]_{\frac{1}{2}}-\left[[\mathrm{Ni}] 4 s^{2} 4 d_{-}\right]_{\frac{3}{2}}$ of Ga-like ions. The central electron temperature associated with each spectrum is shown in each panel.

probably blended with another line of Ge-like ions as mentioned in Sec. II B. The wavelengths of the identified lines are listed in the "LHD expt." column in Table III, where the previously reported wavelengths are indicated by footnotes.

\section{B. EBIT experiment}

We have also carried out a charge state resolved spectral measurement for Eu ions using the Tokyo EBIT [46], taking advantage of the possibility to separate the ionic charge states as described in the rest of this subsection. The experimental setup was similar to that in the previous studies, where a slitless type of grazing incidence flat field spectrometer with a Peltier-cooled CCD was used for observing EUV spectra of $\mathrm{Gd}$ ions $[47,48]$. For producing $\mathrm{Eu}$ ions, a vapor of $\mathrm{Eu}$ was injected into the EBIT from an effusion cell [49]. The temperature of the cell was kept at $400{ }^{\circ} \mathrm{C}$. The electron-beam current and the central magnetic field were $18 \mathrm{~mA}$ and $1 \mathrm{~T}$, respectively. The trapped ions were dumped every $10 \mathrm{~s}$ to prevent unwanted ions, such as tungsten and barium, evaporated from the electron gun cathode, from being accumulated in the trap.

Figure 2 shows the spectra of Eu ions obtained at electron energies of 1.29 to $1.45 \mathrm{keV}$. It is noted that the electron energy refers to the nominal value determined from the potential difference between the electron gun cathode and the middle electrode of the ion trap, i.e., the space charge of the electron beam is not taken into account. Transitions in Ga- and Zn-like $\mathrm{Eu}$, which were identified in previous tokamak observations $[21,50]$, were clearly observed in the region between 10 and $11 \mathrm{~nm}$. It is also confirmed that the spectral features are quite

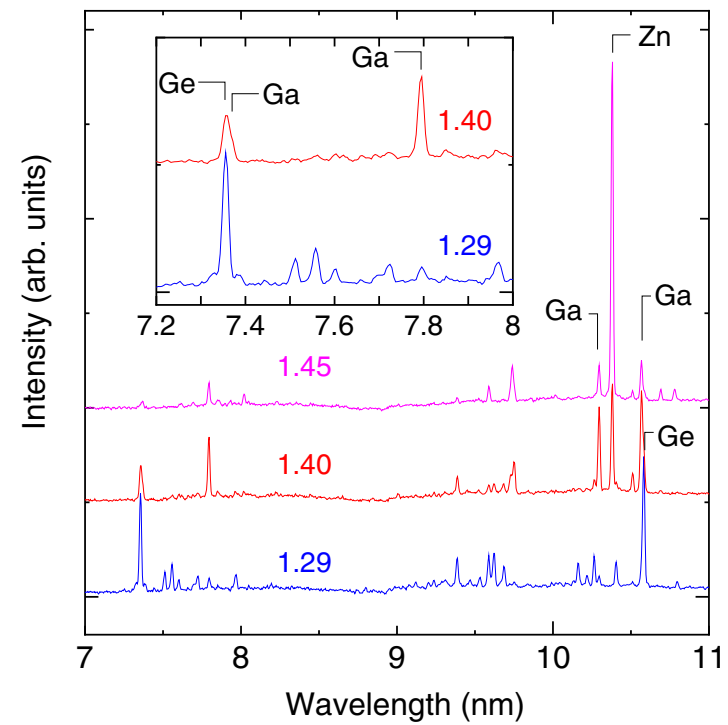

FIG. 2. Electron energy dependence of the EBIT spectra. The numbers indicated on each spectrum represent the electron energy in $\mathrm{keV}$. The notations $\mathrm{Ge}, \mathrm{Ga}$, and $\mathrm{Zn}$ represent the ionic charge states.

similar to those for Ge- to Zn-like Gd obtained with the NISTEBIT at electron energies of 1.378 to $1.520 \mathrm{keV}$ [31]. Thus the prominent lines obtained in the present paper can be assigned as indicated in the figure, and the electron energy suitable for observing Ga-like Eu was determined to be $1.40 \mathrm{keV}$.

The inset of Fig. 2 shows an enlarged view of the wavelength region of current interest. From the energy dependence, the lines at 7.35 and $7.80 \mathrm{~nm}$ can be assigned as emissions from $\mathrm{Ge}$ - and Ga-like Eu, respectively. In addition, a small shoulder was confirmed in the spectrum at $1.40 \mathrm{keV}$, which should be assigned as an emission from Ga-like Eu.

Figure 3(a) shows a high-resolution spectrum of Eu ions at an electron energy of $1.40 \mathrm{keV}$ obtained in the second-order diffraction, in order to resolve the adjacent two lines from Ge- and Ga-like Eu around $7.35 \mathrm{~nm}$. The wavelength range shown in the figure is 14.0 to $16.1 \mathrm{~nm}$ in the first order, which corresponds to 7.0 to $8.05 \mathrm{~nm}$ in the second order, as shown in the upper horizontal axis. As seen in the figure, the small shoulder confirmed in the first-order spectrum shown in Fig. 2 was more clearly resolved.

In order to determine the wavelength of these lines, the wavelength scale was calibrated using nine $\mathrm{W}^{q+}$ lines $(q=$ 37-63) listed in Table I. The $\mathrm{W}$ lines were observed in the first- to third-order diffraction for the first-order wavelength range of 13.7 to $16 \mathrm{~nm}$. Examples of the reference $\mathrm{W}$ spectra are shown in Fig. 3(b), which were obtained with electron energies of 2.49 and $8.49 \mathrm{keV}$. The peak position of each $\mathrm{W}$ line was obtained by unweighted fitting of a Gaussian function to the observed peak. The conversion function from the CCD pixel number to the wavelength was then obtained by fitting a quadratic function to the peak positions of the reference lines, while weighting them by the quadrature sum of the fitting error in the peak position and the uncertainty in the reference wavelength [51]. From the conversion function obtained, the wavelength of the lines $A$ and $B$ in Ga-like Eu was determined to be 7.7933(21) $\mathrm{nm}$ and 7.3691(20) nm, respectively, 


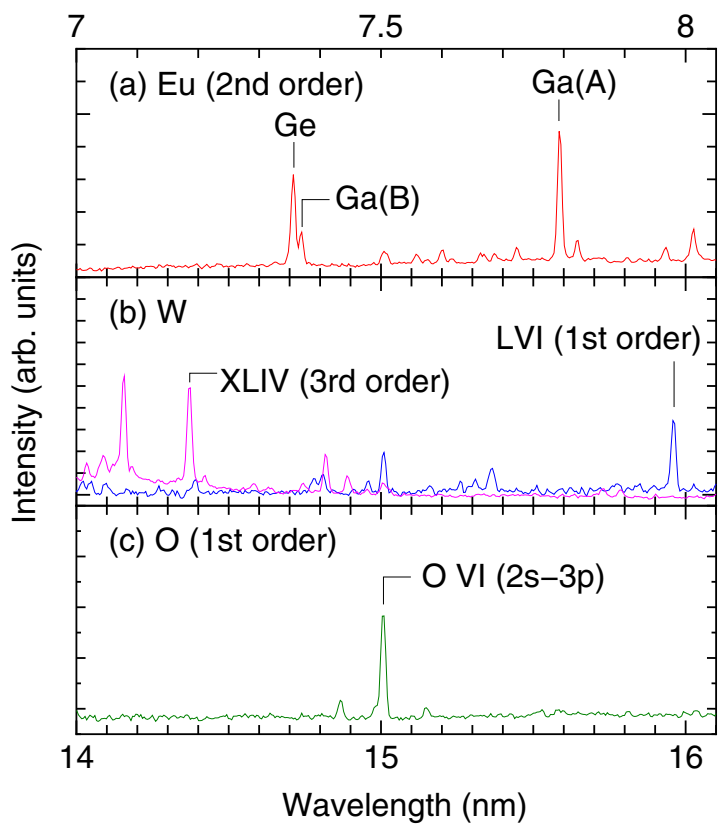

FIG. 3. (a) High resolution spectrum of Eu obtained in the second-order diffraction. The notations $\mathrm{Ge}$ and $\mathrm{Ga}$ represent the ionic charge states. A, [[Ni $\left.] 4 s^{2} 4 p_{-}\right]_{\frac{1}{2}}-\left[[\mathrm{Ni}] 4 s^{2} 4 d_{-}\right]_{\frac{3}{2}}$ transition; B, $\left[[\mathrm{Ni}] 4 s^{2} 4 p_{-}\right]_{\frac{1}{2}}-\left[[\mathrm{Ni}] 4 s 4 p_{+}^{2}\right]_{\frac{3}{2}}$ transition. The upper horizontal scale is the wavelength in the second-order diffraction. (b) W spectra at electron energies of 2.49 (magenta) and $8.49 \mathrm{keV}$ (blue) used in the wavelength calibration. (c) O VI $2 s-3 p$ transitions observed with the same experimental parameters as those used in the observation of the Eu spectrum.

which were identified as $\left[[\mathrm{Ni}] 4 s^{2} 4 p_{-}\right]_{\frac{1}{2}}-\left[[\mathrm{Ni}] 4 s^{2} 4 d_{-}\right]_{\frac{3}{2}}$ and $\left[[\mathrm{Ni}] 4 s^{2} 4 p_{-}\right]_{\frac{1}{2}}-\left[[\mathrm{Ni}] 4 s 4 p_{+}^{2}\right]_{\frac{3}{2}}$ transitions. They are listed in Table III. The spectral line indicated as Ge in Fig. 3 was tentatively identified as the $\left[[\mathrm{Ni}] 4 s^{2} 4 p_{-}^{2}\right]_{0}-\left[[\mathrm{Ni}] 4 s^{2} 4 p_{-} 4 d_{-}\right]_{1}$ transition of Ge-like ions. The uncertainty of the experimental wavelengths was estimated from the following three contributions: (i) the fitting error in the unweighted fitting of a Gaussian to the Eu lines, (ii) the $68 \%$ confidence interval of the conversion function, and (iii) the systematic error estimated as described in the next paragraph. Contributions (i)

TABLE I. W lines used for the wavelength calibration. The wavelengths $\lambda$ were taken from the NIST database [51]. Experimental parameters (diffraction order, electron-beam energy $E_{\mathrm{e}}$, electron-beam current $I_{\mathrm{e}}$, and central magnetic field $B$ ) are also listed.

\begin{tabular}{lccccc}
\hline \hline Charge & $\lambda(\mathrm{nm})$ & Order & $E_{\mathrm{e}}(\mathrm{keV})$ & $I_{\mathrm{e}}(\mathrm{mA})$ & $B(\mathrm{~T})$ \\
\hline $37+$ & $4.5781(5)$ & 3 & 1.84 & 30 & 1.5 \\
$38+$ & $4.6670(4)$ & 3 & 1.84 & 30 & 1.5 \\
$43+$ & $4.7903(5)$ & 3 & 2.49 & 40 & 1.5 \\
$43+$ & $6.0616(6)$ & 3 & 2.49 & 40 & 1.5 \\
$44+$ & $6.0930(5)$ & 3 & 2.49 & 40 & 1.5 \\
$61+$ & $7.404(2)$ & 2 & 10.5 & 100 & 3.0 \\
$63+$ & $7.7700(12)$ & 2 & 10.5 & 100 & 3.0 \\
$62+$ & $7.991(2)$ & 2 & 10.5 & 100 & 3.0 \\
$55+$ & $15.962(3)$ & 1 & 8.49 & 100 & 3.0 \\
\hline \hline
\end{tabular}

TABLE II. The steps of calculations for $[\mathrm{Ni}] 4 s^{2} 4 p J=\frac{1}{2}, \frac{3}{2}$, $[\mathrm{Ni}] 4 s^{2} 4 d J=\frac{3}{2}, \frac{5}{2}$, and $[\mathrm{Ni}] 4 s 4 p^{2} J=\frac{3}{2}, \frac{5}{2}$ states of Ga-like lanthanide atomic ions. The $1 s, 2 s, 2 p, 3 s, 3 p$, and $3 d$ orbitals are treated as core orbitals. The $4 s, 4 p$, and $4 d$ orbitals are treated as valence orbitals. In contrast, $4 f$, and all the $n=5,6$, and 7 orbitals, are treated as correlation orbitals. In steps 1 and 2, all the atomic orbitals are optimized in the SCF iterative procedure. In steps 3 to 6 , only the newly introduced atomic orbitals are optimized in the SCF iterative procedure.

\begin{tabular}{lccc}
\hline \hline No. $^{\mathrm{a}}$ & Step $^{\mathrm{b}}$ & Configurations & CSF $^{\mathrm{c}}$ \\
\hline 2 & 1 & $4 s^{2} 4 p J=1 / 2,3 / 2$ & \\
& & $+4 s^{2} 4 d J=3 / 2,5 / 2$ & 4 \\
3 & 2 & Step $1+4 s 4 p^{2} J=3 / 2,5 / 2$ & 9 \\
4 & 3 & Step $2+4 f$, with v ${ }^{\mathrm{d}}, \mathrm{vv}^{\mathrm{e}}$ & 207 \\
5 & 4 & Step $3+n=5$, with v, vv & 1472 \\
6 & 5 & Step $4+n=6$, with v, vv & 4454 \\
7 & 6 & Step $5+n=7$, with v, vv & 7341 \\
\hline \hline
\end{tabular}

${ }^{\mathrm{a}}$ Index number of numerical calculation

${ }^{\mathrm{b}}$ Order number of steps in the numerical procedure.

${ }^{\mathrm{c}}$ Number of relativistic configurations.

${ }^{\mathrm{d}}$ Single electron excitation of valence orbitals.

${ }^{\mathrm{e}}$ Double electron excitation of valence orbitals.

and (ii) were added in quadrature, while contribution (iii) was linearly added.

The $\mathrm{W}$ lines used in the calibration were observed with experimental parameters (electron energy, electron current, and magnetic field) different from those used in the Eu observation. This could cause a systematic error in the calibrated wavelength scale because the position of the electron beam, which acted as an entrance slit of the spectrometer, could be different between the Eu and W observations. In order to examine this systematic error, the $2 s-3 p$ transitions in O VI were observed with the same experimental parameters that were used in the Eu observation except for the injected gas and the dumping period $(0.2 \mathrm{~s}$ for the $\mathrm{O}$ observation). The observed spectrum is shown in Fig. 3(c). The experimental wavelength of the O VI transition was determined to be $15.0072 \mathrm{~nm}$, whereas the weighted average of the $2 s-3 p_{1 / 2}$ and $2 s-3 p_{3 / 2}$ transitions is known to be $15.0101 \mathrm{~nm}$ [51-53]. The difference between them $(0.0029 \mathrm{~nm})$ was regarded as the systematic error. This corresponds to $0.0014 \mathrm{~nm}$ for the second-order wavelength.

\section{THEORY}

\section{A. Multiconfiguration expansion of atomic states}

By using one of the GRASP family of codes General Purpose Relativistic Atomic Structure Program 92 (GRASP92) [54] with modification, we carry out a set of MCDF calculations for the electronic state structures of lanthanide atomic galliumlike ions (ions with 31 electrons) $\mathrm{La}^{25+}$ to $\mathrm{Lu}^{39+}$. An advantage of those programs is that we can treat the two electron nonlocal exchange interactions without any localpotential approximation [13]. In this context, we can properly evaluate the electron correlations through the interactions between relevant configuration state functions (CSFs) in a sophisticated manner. The optical transitions from the valence 
TABLE III. Wavelengths in nm for $\left[4 s^{2} 4 p_{-}\right]_{\frac{1}{2}}-\left[4 s^{2} 4 d_{-}\right]_{\frac{3}{2}}$ and $\left[4 s^{2} 4 p_{-}\right]_{\frac{1}{2}}-\left[4 s 4 p_{+}^{2}\right]_{\frac{3}{2}}$ transitions of Ga-like lanthanide elements La to Lu. The values without a footnote mark are of the present calculation or experiments. The previously reported LHD data are indicated by footnote marks $b$ and $h$. The uncertainties of LHD data are estimated to be $0.005 \mathrm{~nm}$. The numbers in the round brackets after the EBIT Eu data are the uncertainty estimates.

\begin{tabular}{|c|c|c|c|c|c|c|c|c|c|c|c|}
\hline \multicolumn{2}{|c|}{ Transition } & \multicolumn{5}{|c|}{$\left[4 s^{2} 4 p_{-}\right]_{\frac{1}{2}}-\left[4 s^{2} 4 d_{-}\right]_{\frac{3}{2}}$} & \multicolumn{5}{|c|}{$\left[4 s^{2} 4 p_{-}\right]_{\frac{1}{2}}-\left[4 s 4 p_{+}^{2}\right]_{\frac{3}{2}}$} \\
\hline$Z$ & Ion & $\begin{array}{l}\text { Present } \\
\text { theory }\end{array}$ & $\begin{array}{l}\text { LHD } \\
\text { expt. }\end{array}$ & $\begin{array}{l}\text { EBIT } \\
\text { expt. }\end{array}$ & $\begin{array}{l}\text { Other } \\
\text { expt. }\end{array}$ & $\begin{array}{l}\text { Other } \\
\text { theory }\end{array}$ & $\begin{array}{l}\text { Present } \\
\text { theory }\end{array}$ & $\begin{array}{l}\text { LHD } \\
\text { expt. }\end{array}$ & $\begin{array}{l}\text { EBIT } \\
\text { expt. }\end{array}$ & $\begin{array}{l}\text { Other } \\
\text { expt. }\end{array}$ & $\begin{array}{l}\text { Other } \\
\text { theory }\end{array}$ \\
\hline 57 & $\mathrm{La}$ & 9.765 & 9.827 & & & $9.757^{\mathrm{a}}$ & 10.841 & & & & $10.867^{\mathrm{a}}$ \\
\hline 58 & $\mathrm{Ce}$ & 9.312 & 9.369 & & & $9.307^{\mathrm{a}}$ & 10.221 & 10.304 & & & $10.246^{\mathrm{a}}$ \\
\hline 59 & $\operatorname{Pr}$ & 8.882 & $8.939^{\mathrm{b}}$ & & $8.77^{\mathrm{c}}$ & $\begin{array}{l}8.790^{\mathrm{d}} \\
8.879^{\mathrm{a}} \\
8.776^{\mathrm{e}}\end{array}$ & 9.645 & $9.714^{\mathrm{b}}$ & & & $9.669^{\mathrm{a}}$ \\
\hline 60 & $\mathrm{Nd}$ & 8.472 & $8.526^{\mathrm{b}}$ & $8.5256^{\mathrm{f}}$ & & $8.471^{\mathrm{g}}$ & 9.112 & $9.175^{\mathrm{b}}$ & $9.1761^{\mathrm{f}}$ & & $9.133^{\mathrm{g}}$ \\
\hline 61 & $\mathrm{Pm}$ & 8.077 & & & & $8.079^{\mathrm{g}}$ & 8.619 & & & & $8.638^{g}$ \\
\hline 62 & $\mathrm{Sm}$ & 7.694 & $7.750^{\mathrm{h}}$ & $7.7461^{\mathrm{f}}$ & & $7.650^{\mathrm{g}}$ & 8.167 & $8.215^{\mathrm{h}}$ (blended) & $8.2146^{\mathrm{f}}$ & & $8.182^{\mathrm{g}}$ \\
\hline 63 & $\mathrm{Eu}$ & 7.754 & 7.793 & $7.7933(21)$ & $7.79^{c}$ & $\begin{array}{l}7.242^{\mathrm{d}} \\
7.635^{\mathrm{g}} \\
7.700^{\mathrm{e}}\end{array}$ & 7.319 & 7.367 (blended) & 7.3691(20) & & $\begin{array}{l}7.698^{d} \\
7.766^{g}\end{array}$ \\
\hline 64 & $\mathrm{Gd}$ & 7.379 & 7.411 & $7.413^{\mathrm{f}}$ & $\begin{array}{l}7.41^{\mathrm{c}} \\
7.414^{\mathrm{i}}\end{array}$ & $\begin{array}{l}7.325^{\mathrm{d}} \\
7.388^{\mathrm{g}} \\
7.326^{\mathrm{e}}\end{array}$ & 6.951 & & & & $6.956^{\mathrm{g}}$ \\
\hline 65 & $\mathrm{~Tb}$ & 7.037 & 7.062 & & & $7.043^{\mathrm{g}}$ & 6.593 & & & & $6.608^{g}$ \\
\hline 66 & Dy & 6.721 & 6.745(blended) & $6.750^{\mathrm{f}}$ & $6.75^{\mathrm{c}}$ & $\begin{array}{l}6.668^{\mathrm{d}} \\
6.674^{\mathrm{e}}\end{array}$ & 6.246 & & & & \\
\hline 67 & Ho & 6.427 & 6.455 & & & & 5.914 & & & & \\
\hline 68 & $\mathrm{Er}$ & 6.152 & $6.171^{\mathrm{h}}$ & $6.1755^{\mathrm{f}}$ & & & 5.598 & & & & \\
\hline 69 & $\mathrm{Tm}$ & 5.892 & $5.911^{\mathrm{h}}$ & & & & 5.297 & & & & \\
\hline 70 & $\mathrm{Yb}$ & 5.646 & 5.666 & $5.6694^{\mathrm{f}}$ & $5.74^{\mathrm{c}}$ & $\begin{array}{l}5.597^{\mathrm{d}} \\
5.608^{\mathrm{e}}\end{array}$ & 5.012 & & & & \\
\hline 71 & $\mathrm{Lu}$ & 5.412 & & & & & 4.743 & & & & \\
\hline
\end{tabular}

${ }^{a}$ El-Sayed (MCDF) [60].

${ }^{\mathrm{b}}$ Suzuki et al. (LHD) [30].

${ }^{\mathrm{c}}$ Fournier et al. (Tokamak) [21].

dZilitis (MCDF) [15].

${ }^{\mathrm{e}}$ Fournier et al. (RELAC) [21].

${ }^{f}$ NIST (EBIT) [31,33-36].

gEl-Sayed and Attia (MCDF) [61].

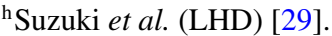

${ }^{\mathrm{i}}$ Fournier et al. (LPP) [21].

shell excited states to the ground states give emissions of EUV radiation. We employed the Relativistic Atomic Transition, Ionization, and Recombination Properties (RATIP) [55] modeling package for the calculation of optical transition strengths; RATIP is a package which works independently of GRASP but requires GRASP wave functions.

We take the following Dirac-Coulomb Hamiltonian $H_{\mathrm{DC}}$ to perform the MCDF procedure:

$$
H_{\mathrm{DC}}=\sum_{i} h_{i}+\sum_{i>j} \frac{1}{r_{i j}},
$$

where $h_{i}$ is the Dirac Hamiltonian of a single electron atomic ion for the $i$ th electron, and $r_{i j}$ is the distance between the electrons $i$ and $j$. We use the atomic units $e=m=\hbar=1$ throughout the paper, unless otherwise specified. In the MCDF calculations, we optimize individual single electron atomic orbitals in atomic state functions (ASFs), $\Psi$, which consist of the linear combination of CSFs, $\Phi_{\alpha}$, numerically by means of the self-consistent field (SCF) iterative procedure, where $\alpha$ is the set of quantum numbers to specify the configuration state [54,56-58]. The Breit interaction and quantum electrodynamic corrections are included as perturbations, using the results of the MCDF calculation [54,56-58].

The ASF $\Psi$ is represented by

$$
\Psi=\sum_{\alpha} c_{\alpha} \Phi_{\alpha}
$$

where $c_{\alpha}$ is the expansion coefficient, which reflects the interactions between the CSFs, and can be called the mixing coefficient of configuration interaction. Both the mixing coefficients $c_{\alpha}$ and the single electron orbitals are optimized simultaneously in the MCDF procedure. We may find the optimum shape of the single electron atomic orbitals that fits with the multiconfiguration state under consideration. We can avoid an excessive expansion of ASFs in terms of many CSFs that contain a number of single electron orbitals; this feature 
gives us a chance to implement realistic physical characteristics in individual single electron orbitals.

In the atomic states of highly charged ions, the ASFs of different but energetically close states $\Psi_{q}$, where $q$ specifies the atomic states, can be expected to have similar forms of single electron atomic orbitals. We may optimize the weighted sum of the ASFs $\Psi_{\text {sum }}$,

$$
\Psi_{\text {sum }}=\sum_{q} w_{q} \Psi_{q},
$$

instead of an individual ASF $\Psi_{q}$, where $w_{q}$ is the weight. In the GRASP family of codes, we call this extended one level (EOL) optimization. The choice of the weights $w_{q}$ is, by virtue of the MCDF method, arbitrary. Normally, we take equal or statistical weights for $w_{q}$. We take $w_{q}=1$ for all $q$, i.e., we take equal weights for a selected set of $\Psi_{q}$ in the present paper. By including both ground- and excited-state ASFs in $\Psi_{\text {sum }}$, we can obtain a common set of single electron atomic orbital functions, which enables us to discuss the electronic state excitations or deexcitations in terms of the change of electron occupations in the atomic orbital set.

By using RATIP [55], we calculate wavelengths and the strengths of electric dipole $(E 1)$ transitions between the ground and excited states. Although we can consider the orbital relaxations in RATIP, we adopt the common basis orbital sets in both the states before and after the optical transition. The nonorthogonality problem is not serious for $E 1$ transitions in highly charged ions. Further, by using the common basis orbitals, we can avoid the risk of facing large errors in the energy offset that may arise from the deep core electrons.

\section{B. Numerical calculation}

We consider the Ga-like electronic ground states $4 s^{2} 4 p J=\frac{1}{2}, \frac{3}{2}$, valence excited states $4 s^{2} 4 d J=\frac{3}{2}, \frac{5}{2}$, and subvalence excited states $4 s 4 p^{2} J=\frac{3}{2}, \frac{5}{2}$ in the same MCDF procedure with the EOL option in GRASP92 [54]. Here, in this and the following sections, we omit the core configuration notation $\mathrm{Ni}$ for brevity. We optimize the single electron atomic orbitals stepwise [59], including higher principal quantum number orbitals up to $n=7$. In Table II, we show the calculation steps and the number of relativistic CSFs in their respective MCDF procedures. In step 1, we consider only the minimal set of electronic configurations for the ground states $4 s^{2} 4 p J=\frac{1}{2}, \frac{3}{2}$ and the excited states $4 s^{2} 4 d J=\frac{3}{2}, \frac{5}{2}$, and they are optimized simultaneously in the MCDF procedure. In step 2, we include $4 s$ excited-state configurations $4 s 4 p^{2} J=\frac{3}{2}, \frac{5}{2}$ and all atomic orbitals are simultaneously optimized in the MCDF procedure. In steps 3 through 6 , we successively include $4 f$, all the orbitals with the principal quantum number $n=5,6$, and 7 as correlation orbitals. In each step from 3 to 6 , only the newly included atomic orbitals are optimized in the MCDF procedure, while the others are fixed.

For each step of the calculations we also calculate the wavelengths of the optical transitions and their Einstein $A$ coefficients using RATIP [55]. In Fig. 4, we show, as an example, the convergence feature of transition wavelengths and Einstein $A$ coefficients for Ga-like $\mathrm{Gd}^{33+}$ ions. We can observe that they are well converged at calculation number 6 , i.e., at
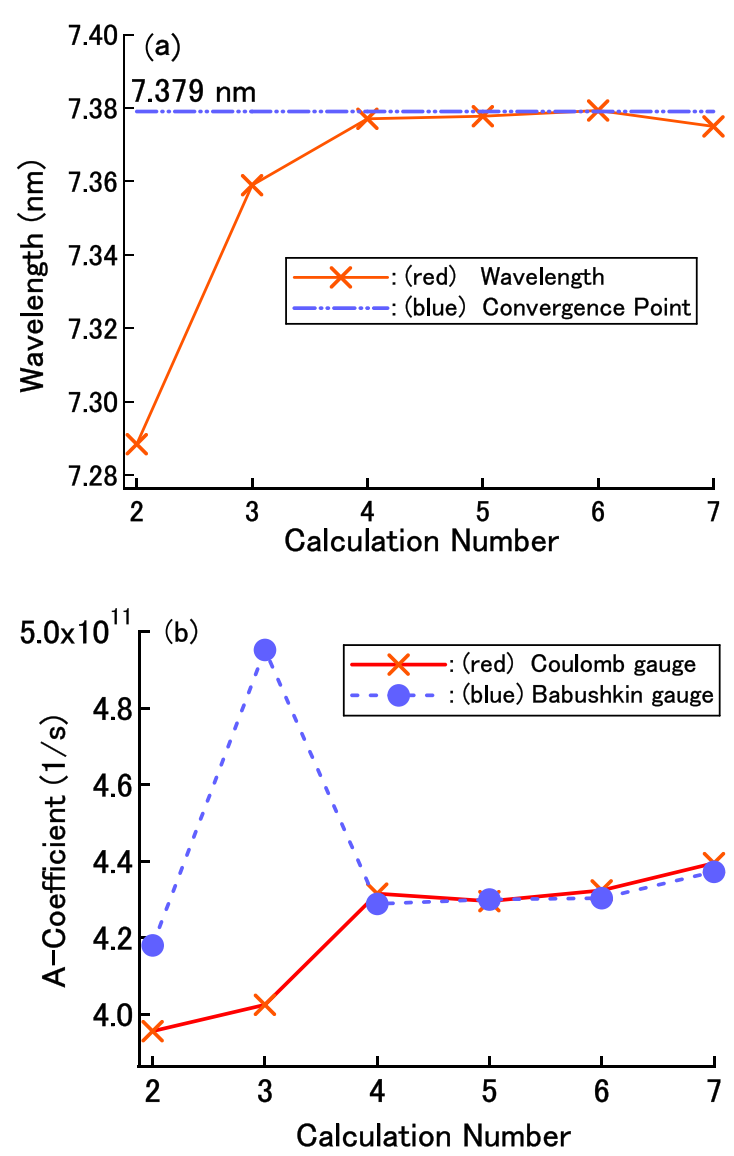

FIG. 4. Convergence feature of the $\left[4 s^{2} 4 p_{-}\right]_{\frac{1}{2}}-\left[4 s^{2} 4 d_{-}\right]_{\frac{3}{2}}$ wavelength and Einstein $A$ coefficient for Ga-like $\mathrm{Gd}$ ions $\left(\mathrm{Gd}^{33+}\right)$. (a) Calculation number dependence of calculated wavelength. (b) Calculation number dependence of $A$ coefficients in Coulomb gauge (corresponding to velocity form) and Babushkin gauge (corresponding to length form).

calculation step 5. The calculated wavelength is $7.379 \mathrm{~nm}$, whereas the corresponding value of the LHD experiment is $7.411 \mathrm{~nm}$ as found in Table III. They agree within a difference of $0.43 \%$. We use the values at calculation number 6 for all the ion species in the present paper.

In Table III, we give the calculated wavelengths, the results of present LHD and EBIT experiments in $\mathrm{nm}$ for transitions $\left[4 s^{2} 4 p_{-}\right]_{\frac{1}{2}}-\left[4 s^{2} 4 d_{-}\right]_{\frac{3}{2}}$ and $\left[4 s^{2} 4 p_{-}\right]_{\frac{1}{2}}-\left[4 s 4 p_{+}^{2}\right]_{\frac{3}{2}}$. We have also listed in the table available theoretical as well as experimental values in the literature. We find that the differences between the present calculation and both the LHD and EBIT experiments for $\left[4 s^{2} 4 p_{-}\right]_{\frac{1}{2}}-\left[4 s^{2} 4 d_{-}\right]_{\frac{3}{2}}$ transitions are mostly less than $0.50 \%$, except $0.61 \%$ for $\mathrm{Ce}, 0.63 \%$ for $\mathrm{Nd}$, and $0.72 \%$ for Sm. Although the number of experimental values is small, the differences between the present theory and both the LHD and EBIT experiments are found to be $0.81 \%$ for Ce and less for the $\left[4 s^{2} 4 p_{-}\right]_{\frac{1}{2}}-\left[4 s 4 p_{+}^{2}\right]_{\frac{3}{2}}$ transition. For all the values compared, the present theoretical wavelengths are systematically shorter than found in the LHD and EBIT experiments. This might be due to the omission of core-valence virtual excitation in the evaluation of correlation effects in the present MCDF procedure, and could be a subject for further investigations 


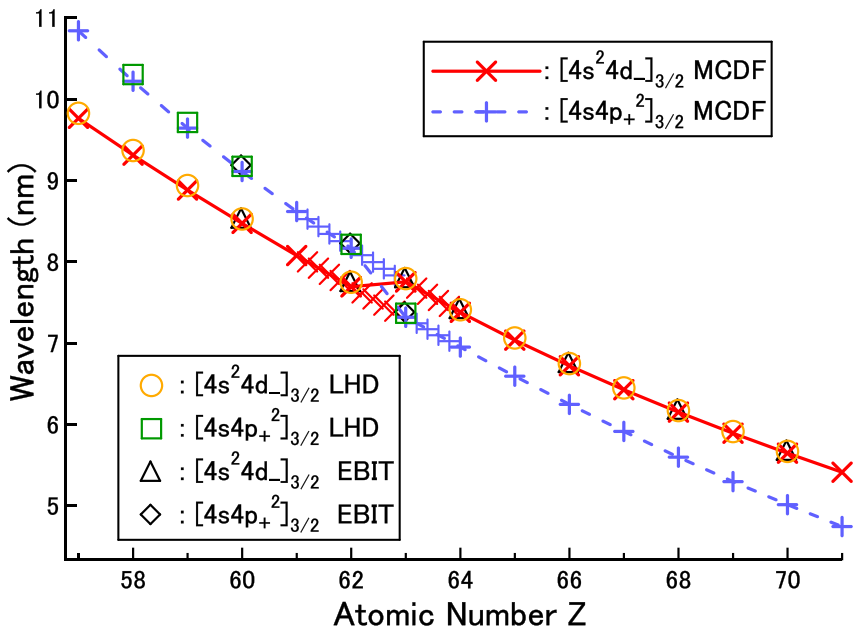

FIG. 5. Wavelength for the transitions $\left[4 s^{2} 4 p_{-}\right]_{\frac{1}{2}}-\left[4 s^{2} 4 d_{-}\right]_{\frac{3}{2}}$ and $\left[4 s^{2} 4 p_{-}\right]_{\frac{1}{2}}-\left[4 s 4 p_{+}^{2}\right]_{\frac{3}{2}}$ of Ga-like ions of lanthanide elements with $Z=57-71$ (La to Lu). Solid (red) line and symbol x, present MCDF calculation; open (orange) circle, LHD experiment; open (black) triangle, EBIT experiment for the $\left[4 s^{2} 4 p_{-}\right]_{1}-\left[4 s^{2} 4 d_{-}\right]_{3}$ transition. Broken (blue) line and symbol + , present MCDF calculation; open (green) square, LHD experiment; open (black) diamond, EBIT experiment for the $\left[4 s^{2} 4 p_{-}\right]_{\frac{1}{2}}-\left[4 s 4 p_{+}^{2}\right]_{\frac{3}{2}}$ transition.

in forthcoming papers. In fact, $\mathrm{Hu}$ et al. [62] have reported $0.2 \%$ of core-valence and core-core contributions for similar transitions in Ga-like $\mathrm{Yb}$ ions.

We can further point out in Table III that there are anomalous behaviors in the $Z$ dependence of the wavelengths near $Z=62$ to 63 . The wavelength of the $\operatorname{Eu}(Z=63)$ $\left[4 s^{2} 4 p_{-}\right]_{\frac{1}{2}}-\left[4 s^{2} 4 d_{-}\right]_{\frac{3}{2}}$ line, $7.754 \mathrm{~nm}$, is longer than the value $7.694 \mathrm{~nm}$ of the neighboring lighter element $\operatorname{Sm}(Z=62)$, while the wavelength of the $\left[4 s^{2} 4 p_{-}\right]_{\frac{1}{2}}-\left[4 s 4 p_{+}^{2}\right]_{\frac{3}{2}}$ line shows an abrupt decrease from $Z=62$ to 63 . This behavior can be observed clearly in Fig. 5. We have plotted in Fig. 5 the presently observed and calculated wavelengths of $\left[4 s^{2} 4 p_{-}\right]_{\frac{1}{2}}-\left[4 s^{2} 4 d_{-}\right]_{\frac{3}{2}}$ and $\left[4 s^{2} 4 p_{-}\right]_{\frac{1}{2}}-\left[4 s 4 p_{+}^{2}\right]_{\frac{3}{2}}$ transitions, together with the previously obtained LHD and EBIT data. Between $Z=61$ and 64, we have also plotted the theoretical wavelengths for noninteger atomic numbers; the GRASP family of codes [54,56-58] allows for calculations with noninteger $Z$. We normally name the energy level according to the leading configuration in the atomic state as a convention, and the leading configurations in the energetically lower and upper states change between $Z=62$ and 63 (see Table IV). We can find that the two $Z$-dependent wavelength curves are crossing between $Z=62$ and 63. However, it must be pointed out that the determination of the crossing point is not trivial, since due to the configuration interaction the ASFs are mixtures of CSFs $\left[4 s^{2} 4 d_{-}\right]_{\frac{3}{2}}$ and $\left[4 s 4 p_{+}^{2}\right]_{\frac{3}{2}}$, and the percentage fractions of the CSF components are quite sensitive to the procedure of calculation. We will discuss those points in detail in Sec. IV.

In Fig. 6, we show the $Z$ dependence of Einstein $A$ coefficients for the $\left[4 s^{2} 4 p_{-}\right]_{\frac{1}{2}}-\left[4 s^{2} 4 d_{-}\right]_{\frac{3}{2}}$ and $\left[4 s^{2} 4 p_{-}\right]_{\frac{1}{2}}-$ $\left[4 s 4 p_{+}^{2}\right]_{\frac{3}{2}}$ transitions. In the vicinity of $Z=63$, configurations $\left[4 s^{2} 4 d_{-}\right]_{\frac{3}{2}}$ and $\left[4 s 4 p_{+}^{2}\right]_{\frac{3}{2}}$ are almost equally mixed in both the atomic states $\left[4 s^{2} 4 d_{-}\right]_{\frac{3}{2}}$ and $\left[4 s 4 p_{+}^{2}\right]_{\frac{3}{2}}$ (see Table IV). The radiative properties can be also expected to be similar in both atomic states; the originally optically forbidden atomic state $\left[4 s 4 p_{+}^{2}\right]_{\frac{3}{2}}$ may become active for dipole transition due to the contribution of the radiative intensities from the $\left[4 s^{2} 4 d_{-}\right]_{\frac{3}{2}}$ configuration that is mixed in the atomic state $\left[4 s 4 p_{+}^{2}\right]_{\frac{3}{2}}$. It can be suggested that the spectral intensities

TABLE IV. The occupation percentage fractions of configurations in the atomic states $\left[4 s^{2} 4 d_{-}\right]_{\frac{3}{2}}$ (left) and $\left[4 s 4 p_{+}^{2}\right]_{\frac{3}{2}}\left(\right.$ right): $\left|c_{\alpha}\right|^{2} \times 100$ [see Eq. (2)]. The columns labeled Third CSF give percentages of third leading CSFs and the columns labeled Configuration represent their configuration.

\begin{tabular}{|c|c|c|c|c|c|c|c|c|c|}
\hline \multirow[b]{2}{*}{$Z$} & \multirow[b]{2}{*}{ Ion } & \multirow[b]{2}{*}[4s^{2}4d_{-}]{$_{\frac{3}{2}}$} & \multicolumn{2}{|c|}{$\left[4 s^{2} 4 d_{-}\right]_{\frac{3}{2}}$} & \multirow[b]{2}{*}{ Configuration } & \multirow[b]{2}{*}[4s4p_{+}^{2}]{$_{\frac{3}{2}}$} & \multicolumn{2}{|c|}{$\left[4 s 4 p_{+}^{2}\right]_{\frac{3}{2}}$} & \multirow[b]{2}{*}{ Configuration } \\
\hline & & & $\overline{\left[4 s 4 p_{+}^{2}\right]_{\frac{3}{2}}}$ & $\overline{\text { Third CSF }}$ & & & $\overline{\left[4 s^{2} 4 d_{-}\right]_{\frac{3}{2}}}$ & Third CSF & \\
\hline 57 & $\mathrm{La}$ & 85.6 & 10.7 & 1.7 & {$\left[\left(4 s 4 p_{-}\right)_{0} 4 p_{+}\right]_{\frac{3}{7}}$} & 80.1 & 8.3 & 9.8 & {$\left[\left(4 s 4 p_{-}\right)_{1} 4 p_{+}\right]_{\frac{3}{7}}$} \\
\hline 58 & $\mathrm{Ce}$ & 83.6 & 12.9 & 1.6 & {$\left[\left(4 s 4 p_{-}\right)_{0} 4 p_{+}\right]_{\frac{3}{2}}^{2}$} & 78.8 & 10.5 & 8.9 & {$\left[\left(4 s 4 p_{-}\right)_{1} 4 p_{+}\right]_{\frac{3}{2}}^{2}$} \\
\hline 59 & $\operatorname{Pr}$ & 80.2 & 16.5 & 1.5 & {$\left[\left(4 s 4 p_{-}\right)_{0} 4 p_{+}\right]_{\frac{3}{2}}$} & 75.9 & 14.0 & 8.4 & {$\left[\left(4 s 4 p_{-}\right)_{1} 4 p_{+}\right]_{\frac{3}{2}}^{2}$} \\
\hline 60 & $\mathrm{Nd}$ & 75.3 & 21.6 & 1.4 & {$\left[\left(4 s 4 p_{-}\right)_{0} 4 p_{+}\right]_{\frac{3}{2}}^{2}$} & 71.4 & 18.9 & 7.9 & {$\left[\left(4 s 4 p_{-}\right)_{1} 4 p_{+}\right]_{\frac{3}{2}}^{2}$} \\
\hline 61 & $\mathrm{Pm}$ & 68.2 & 29.0 & 1.3 & {$\left[\left(4 s 4 p_{-}\right)_{0} 4 p_{+}\right]_{\frac{3}{2}}^{2}$} & 64.6 & 26.1 & 7.4 & {$\left[\left(4 s 4 p_{-}\right)_{1} 4 p_{+}\right]_{\frac{3}{2}}^{2}$} \\
\hline 62 & $\mathrm{Sm}$ & 58.5 & 38.9 & 1.1 & {$\left[\left(4 s 4 p_{-}\right)_{0} 4 p_{+}\right]_{\frac{3}{2}}^{2}$} & 55.2 & 35.9 & 6.9 & {$\left[\left(4 s 4 p_{-}\right)_{1} 4 p_{+}\right]_{\frac{3}{2}}^{2}$} \\
\hline 63 & $\mathrm{Eu}$ & 47.7 & 43.8 & 6.3 & {$\left[\left(4 s 4 p_{-}\right)_{1} 4 p_{+}\right]_{\frac{3}{2}}^{2}$} & 50.8 & 46.8 & 0.9 & {$\left[\left(4 s 4 p_{-}\right)_{0} 4 p_{+}\right]_{\frac{3}{3}}^{2}$} \\
\hline 64 & $\mathrm{Gd}$ & 59.8 & 32.3 & 5.6 & {$\left[\left(4 s 4 p_{-}\right)_{1} 4 p_{+}\right]_{\frac{3}{2}}^{2}$} & 62.8 & 34.8 & 0.6 & {$\left[\left(4 s 4 p_{-}\right)_{0} 4 p_{+}\right]_{\frac{3}{2}}^{2}$} \\
\hline 65 & $\mathrm{~Tb}$ & 69.9 & 22.6 & 4.9 & {$\left[\left(4 s 4 p_{-}\right)_{1} 4 p_{+}\right]_{\frac{3}{2}}$} & 72.8 & 24.7 & 0.8 & {$\left[\left(4 s 4 p_{-}\right)_{1} 4 p_{+}\right]_{\frac{3}{2}}^{2}$} \\
\hline 66 & Dy & 77.4 & 15.6 & 4.4 & {$\left[\left(4 s 4 p_{-}\right)_{1} 4 p_{+}\right]_{\frac{3}{2}}$} & 80.2 & 17.3 & 1.0 & {$\left[\left(4 s 4 p_{-}\right)_{1} 4 p_{+}\right]_{\frac{3}{2}}^{2}$} \\
\hline 67 & Ho & 82.5 & 10.8 & 3.9 & {$\left[\left(4 s 4 p_{-}\right)_{1} 4 p_{+}\right]_{\frac{3}{2}}^{2}$} & 85.3 & 12.2 & 1.1 & {$\left[\left(4 s 4 p_{-}\right)_{1} 4 p_{+}\right]_{\frac{3}{2}}^{2}$} \\
\hline 68 & $\mathrm{Er}$ & 85.9 & 7.6 & 3.6 & {$\left[\left(4 s 4 p_{-}\right)_{1} 4 p_{+}\right]_{\frac{3}{2}}^{2}$} & 88.7 & 8.8 & 1.2 & {$\left[\left(4 s 4 p_{-}\right)_{1} 4 p_{+}\right]_{\frac{3}{2}}^{2}$} \\
\hline 69 & $\mathrm{Tm}$ & 88.3 & 5.6 & 3.3 & {$\left[\left(4 s 4 p_{-}\right)_{1} 4 p_{+}\right]_{\frac{3}{2}}^{2}$} & 91.1 & 6.5 & 1.2 & {$\left[\left(4 s 4 p_{-}\right)_{1} 4 p_{+}\right]_{\frac{3}{3}}^{2}$} \\
\hline 70 & $\mathrm{Yb}$ & 89.9 & 4.1 & 3.1 & {$\left[\left(4 s 4 p_{-}\right)_{1} 4 p_{+}\right]_{\frac{3}{2}}^{2}$} & 92.7 & 4.9 & 1.2 & {$\left[\left(4 s 4 p_{-}\right)_{1} 4 p_{+}\right]_{\frac{3}{2}}^{2}$} \\
\hline 71 & $\mathrm{Lu}$ & 91.1 & 3.2 & 3.0 & {$\left[\left(4 s 4 p_{-}\right)_{1} 4 p_{+}\right]_{\frac{3}{2}}$} & 94.0 & 3.8 & 1.2 & {$\left[\left(4 s 4 p_{-}\right)_{1} 4 p_{+}\right]_{\frac{3}{2}}^{2}$} \\
\hline
\end{tabular}




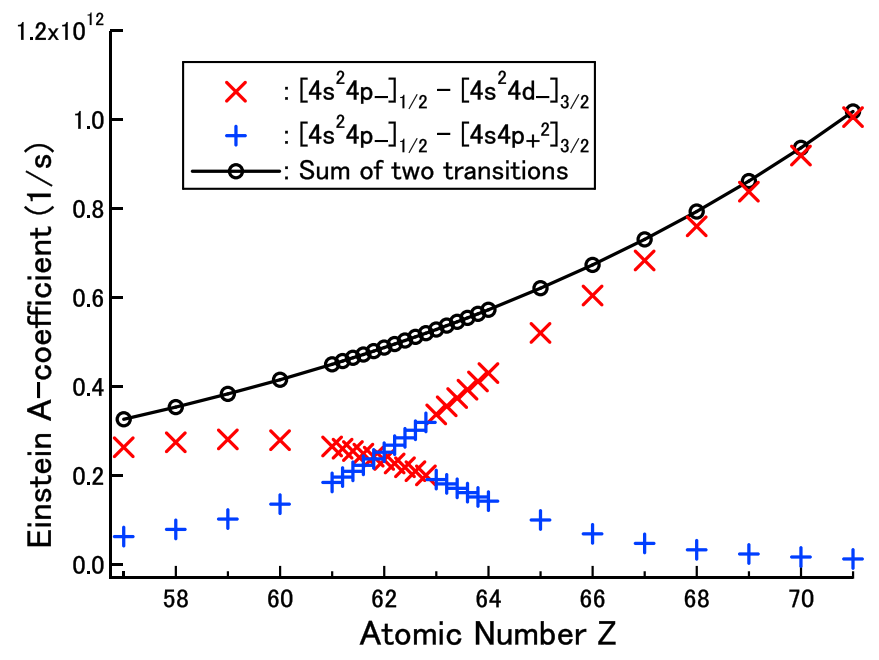

FIG. 6. $Z$ dependence of Einstein $A$ coefficients for $\left[4 s^{2} 4 p_{-}\right]_{\frac{1}{2}}-\left[4 s^{2} 4 d_{-}\right]_{\frac{3}{2}} \quad[(\mathrm{red}) \quad$ cross $]$ and $\left[4 s^{2} 4 p_{-}\right]_{\frac{1}{2}}-\left[4 s 4 p_{+}^{2}\right]_{\frac{3}{2}}$ [(blue) plus] transitions. The (black) solid line with open circles represents the sum of two transitions.

of both $\left[4 s^{2} 4 p_{-}\right]_{\frac{1}{2}}-\left[4 s^{2} 4 d_{-}\right]_{\frac{3}{2}}$ and $\left[4 s^{2} 4 p_{-}\right]_{\frac{1}{2}}-\left[4 s 4 p_{+}^{2}\right]_{\frac{3}{2}}$ transitions are more or less the same in this region if the state distribution is statistical.

\section{DISCUSSION}

As illustrated in Sec. II, the observed experimental spectra consist of lines from ions with different charge states. The assignment of the spectral lines is carried out by comparison to theory, and by taking advantage of the $Z$ dependence of the line emission. In real atomic systems, atomic states are a mixture of configurations with the same symmetry, due to possible configuration interaction. The atomic states are normally assigned according to the names of the leading configurations. However, the mixing of configurations often makes assignments challenging.

In Table IV, we list the occupation percentage fractions $\left|c_{\alpha}\right|^{2} \times 100$ [see Eq. (2)] of the leading three CSFs in the excited-state ASFs. For the two excited states, we have assigned their terms according to the CSFs with the largest percentage fractions. We find in Table IV that a fraction of the leading configuration hits a minimum at $Z=63(\mathrm{Eu})$ for both atomic states $\left[4 s^{2} 4 d_{-}\right]_{\frac{3}{2}}$ and $\left[4 s 4 p_{+}^{2}\right]_{\frac{3}{2}}$. In Fig. 5, We find that the order of their wavelengths changes from $Z=62$ to 63. To understand this level crossing feature, we here consider a mixing angle $\theta$. As noted by Nakamura et al. [17], an ASF with two CSFs can be represented by a rotation in the two-dimensional configuration space. As seen in Table IV the present excited atomic states mostly consist of the two configurations $\alpha \equiv\left[4 s^{2} 4 d_{-}\right]_{\frac{3}{2}}$ and $\beta \equiv\left[4 s 4 p_{+}^{2}\right]_{\frac{3}{2}}$. We take $\Phi_{\alpha}$ and $\Phi_{\beta}$ as the corresponding CSFs. We take $\Psi_{\mathrm{H}}$ and $\Psi_{\mathrm{L}}$ as the two ASFs with higher and lower energies, respectively. Then we have

$$
\left(\begin{array}{l}
\Psi_{\mathrm{H}} \\
\Psi_{\mathrm{L}}
\end{array}\right) \simeq\left(\begin{array}{cc}
\cos \theta & \sin \theta \\
-\sin \theta & \cos \theta
\end{array}\right)\left(\begin{array}{l}
\Phi_{\alpha} \\
\Phi_{\beta}
\end{array}\right)
$$

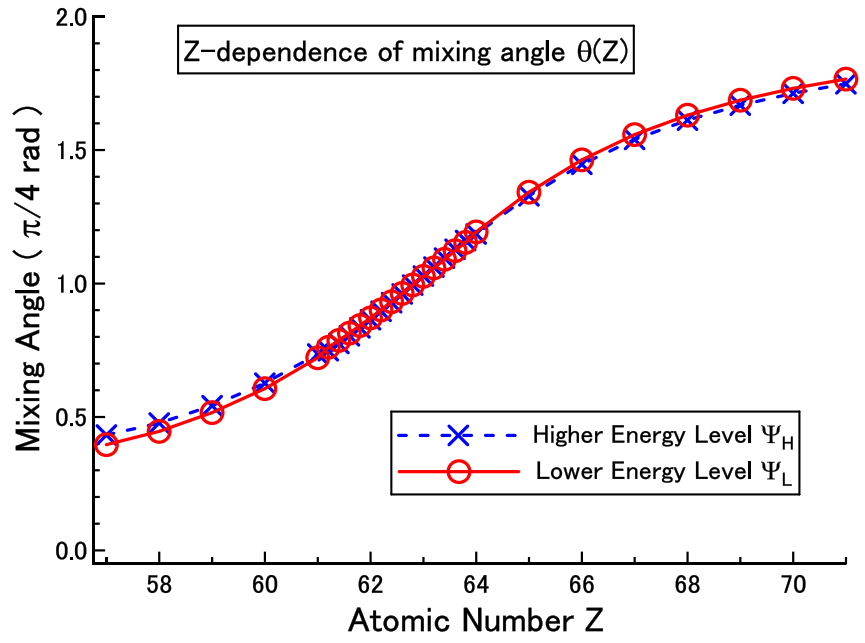

FIG. 7. $Z$ dependence of mixing angle $\theta(Z)$ in units of $\pi / 4 \mathrm{rad}$. Broken line with cross (blue), $\Psi_{\mathrm{H}}$; solid line with open circle (red), $\Psi_{\mathrm{L}}$ [see Eqs. (4) and (5) for their definition].

The mixing angle $\theta$ can be calculated by the relation

$$
\theta=\arctan \left(\frac{\sqrt{\left|c_{\beta}\right|^{2}}}{\sqrt{\left|c_{\alpha}\right|^{2}}}\right)
$$

for each $Z$ referring to the data in Table IV and the data for fractional $Z$ from 61.2 to 63.8. In Fig. 7, we plot $\theta(Z)$ for $\Psi_{\mathrm{H}}$ and $\Psi_{\mathrm{L}}$. They are slightly different because of the contribution of minor components. We find that $\theta(Z)$ is a smooth function of $Z$. We can see that the curve $\theta(Z)$ hits $\pi / 4$ at $Z=62.8$, where the leading CSFs in $\Psi_{\mathrm{H}}$ and $\Psi_{\mathrm{L}}$ change. In other words the atomic states $\Psi_{\mathrm{H}}$ and $\Psi_{\mathrm{L}}$ exchange their leading configurations here. Because we conventionally name the atomic state by the name of the leading configuration, we can say that the level crossing takes place between $Z=62$ and 63 .

Next we can point out that the change of the $\left[4 s 4 p_{+}^{2}\right]_{\frac{3}{2}}$ configuration to the ground-state $\left[4 s^{2} 4 p_{-}\right]_{\frac{1}{2}}$ configuration requires two electron changes from $4 p_{+}$to $4 s$ and $4 p_{+}$to $4 p_{-}$orbitals, whereas the $\left[4 s^{2} 4 d_{-}\right]_{\frac{3}{2}}$ configuration requires only a single electron change from $4 d_{-}$to $4 p_{-}$. The configuration $\left[4 s 4 p_{+}^{2}\right]_{\frac{3}{2}}$ is dipole forbidden for transition to the ground-state configuration $\left[4 s^{2} 4 p_{-}\right]_{\frac{1}{2}}$, whereas $\left[4 s^{2} 4 d_{-}\right]_{\frac{3}{2}}$ is dipole allowed. As found in Table IV, the ASF of the atomic state $\left[4 s 4 p_{+}^{2}\right]_{\frac{3}{2}}$ is a linear combination of configurations $\left[4 s 4 p_{+}^{2}\right]_{\frac{3}{2}}$ and $\left[4 s^{2} 4 d_{-}\right]_{\frac{3}{2}}$. The atomic state $\left[4 s 4 p_{+}^{2}\right]_{\frac{3}{2}}$ then is made visible by the contribution of the intensity from the dipole transition between the configurations $\left[4 s^{2} 4 d_{-}\right]_{\frac{3}{2}}$ and [ $\left.4 s^{2} 4 p_{-}\right]_{\frac{1}{2}}$. In Fig. 6 we plotted the sum of $A$ coefficients for two transitions in a solid (black) line with open circles. It is found out that the curve fits well with the function of the fourth power of an effective atomic number: $0.88 \times 10^{4}(Z-$ $13.2)^{4}\left(\mathrm{~s}^{-1}\right)$. It is known that the $A$ coefficient obeys the fourth power of the effective atomic number $[63,64]$. We can understand that both spectral lines are made visible mostly from the intensity of the $\left[4 s^{2} 4 d_{-}\right]_{\frac{3}{2}}$ configuration that is contributing to both atomic states. As seen in Fig. 6, the $A$ coefficient for the $\left[4 s^{2} 4 p_{-}\right]_{\frac{1}{2}}-\left[4 s 4 p_{+}^{2}\right]_{\frac{3}{2}}$ transition becomes maximum 

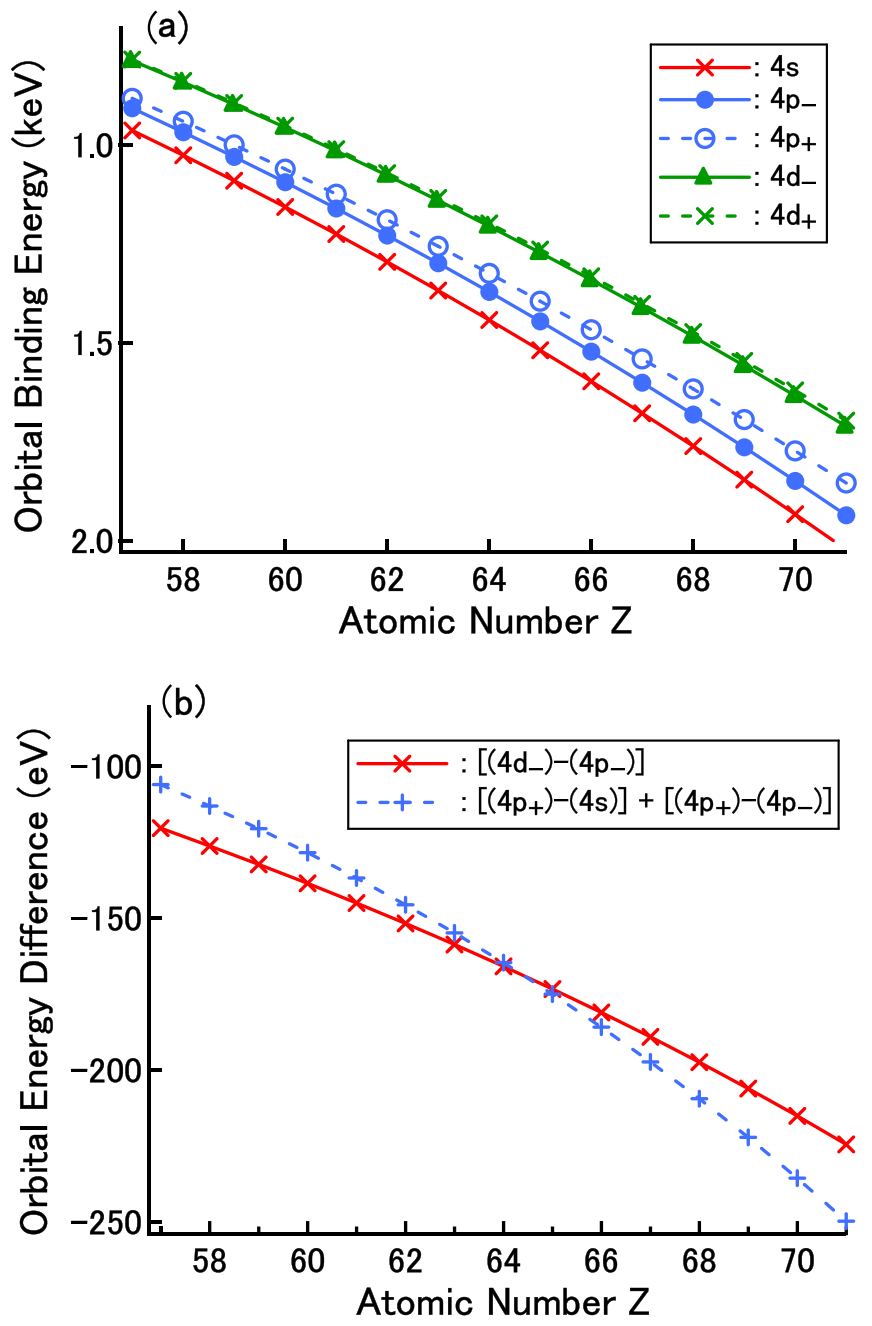

FIG. 8. (a) $Z$ dependence of atomic orbital binding energies in units of keV. (b) $Z$ dependence of atomic orbital energy differences in units of $\mathrm{eV}$.

at $Z=62$, and it is comparable with that of the $\left[4 s^{2} 4 p_{-}\right]_{\frac{1}{2}}-$ $\left[4 s^{2} 4 d_{-}\right]_{\frac{3}{2}}$ transition near $Z=62$. There is a good reason that we observe a line for the $\left[4 s^{2} 4 p_{-}\right]_{\frac{1}{2}}-\left[4 s 4 p_{+}^{2}\right]_{\frac{3}{2}}$ transition near the level crossing region. As found in Fig. 3, we clearly observed for Eu ions two spectral peaks, $A$ and $B$, although the intensity ratio is different from the calculation. Detailed population analysis of the excited states in the plasma would be needed for further understanding.

Now, on the other hand, the energy separation between $4 p_{-}$and $4 p_{+}$orbitals increases as $Z$ increases, due to the so called spin-orbit interaction. Along with the increase of $Z$, the energy difference $\left[4 s 4 p_{+}^{2}\right]_{\frac{3}{2}}-\left[4 s^{2} 4 p_{-}\right]_{\frac{1}{2}}$ is enhanced and overtakes the energy difference $\left[4 s^{2} 4 d_{-}\right]_{\frac{3}{2}}-\left[4 s^{2} 4 p_{-}\right]_{\frac{1}{2}}$ at some value of $Z$, if it is larger than the former at the low- $Z$ end. We may observe the effect as the crossing of transition energy or wavelength curves. By performing fully relativistic calculations on the energy levels and dynamics of the atomic systems, we may resolve if the crossing takes place in the $Z$ range of lanthanide elements. In Fig. 8, $Z$ dependence of the atomic orbital energies is plotted in Fig. 8(a), and the energy differences between $4 d_{-}$and $4 p_{-}$orbitals and the sum of energy differences between $4 p_{+}$and $4 s$ orbitals and between $4 p_{+}$and $4 p_{-}$orbitals are also plotted in Fig. 8(b). We find in Fig. 8(b) that two curves cross between $Z=64$ and 65 . Because we are considering highly charged heavy atomic systems, we can expect that these energy curves roughly simulate the transition energies of respective states in the absence of electron correlations. Due to the electron correlations, the actual crossing point has been shifted from between $Z=64$ and 65 to between $Z=62$ and 63 , as pointed out in the preceding discussions (see Fig. 5).

The spin-orbit interaction in $4 p_{-}$and $4 p_{+}$atomic orbitals plays the most important role for level crossings between $\left[4 s^{2} 4 d_{-}\right]_{\frac{3}{2}}$ and $\left[4 s 4 p_{+}^{2}\right]_{\frac{3}{2}}$ states along the increase of the atomic number $Z$. The role of the spin-orbit interactions on the level crossings was pointed out by Kato et al. [16] in the x-ray emission spectral lines of highly charged Ne-like atomic ions with atomic numbers 50 through 56. In the present paper, we observed crossings between the levels with a higher principal quantum number $n=4$, by means of a comparison between accurate spectral measurements and precise ab initio atomic structure calculations.

\section{CONCLUSION}

EUV optical emission lines were studied in detail for $\left[4 s^{2} 4 p_{-}\right]_{\frac{1}{2}}-\left[4 s^{2} 4 d_{-}\right]_{\frac{3}{2}}$ and $\left[4 s^{2} 4 p_{-}\right]_{\frac{1}{2}}-\left[4 s 4 p_{+}^{2}\right]_{\frac{3}{2}}$ transitions of Ga-like $\mathrm{La}$ to $\mathrm{Lu}$ atomic ions. They were spectroscopically measured in LHD and Tokyo EBIT. The agreement of the wavelengths between both experiments was very good (within $0.03 \%$ ). The $\mathrm{Eu}^{32+}\left[4 s^{2} 4 p_{-}\right]_{\frac{1}{2}}-\left[4 s 4 p_{+}^{2}\right]_{\frac{3}{2}}$ line was confirmed by the charge separated EBIT experiment.

Due to the increase of spin-orbit splitting between $4 p_{-}$ and $4 p_{+}$orbital energies along with the increase of $Z$, the order of level energies of $\left[4 s^{2} 4 d_{-}\right]_{\frac{3}{2}}$ and $\left[4 s 4 p_{+}^{2}\right]_{\frac{3}{2}}$ is reversed from $\mathrm{La}(Z=57)$ to $\mathrm{Lu}(Z=71)$, causing the crossing of $Z$-dependence curves of energies or wavelengths for their optical transitions to the ground state. The atomic number $Z$ dependence of spectral line positions and strengths was theoretically calculated by means of the MCDF approximations. The interaction of $\left[4 s^{2} 4 d_{-}\right]_{\frac{3}{2}}$ and $\left[4 s 4 p_{+}^{2}\right]_{\frac{3}{2}}$ configurations causes their mixing in corresponding atomic states. The originally optically forbidden $\left[4 s^{2} 4 p_{-}\right]_{\frac{1}{2}}-\left[4 s 4 p_{+}^{2}\right]_{\frac{3}{2}}$ line is made visible by the contribution of the $\left[4 s^{2} 4 p_{-}\right]_{\frac{1}{2}}-\left[4 s^{2} 4 d_{-}\right]_{\frac{3}{2}}$ transition near the level crossing region. By a detailed analysis of leading configurations based on the MCDF calculations, we made the level assignment. The levels were found to cross between $Z=62$ and 63, whereas Zilitis [15] pointed out that it was between $Z=63$ and 64. It would be desirable for the present paper to promote an interest in atomic physics using LHD or other nuclear fusion devices.

\section{ACKNOWLEDGMENTS}

The authors acknowledge the LHD experimental group for their assistance. This work was also partially funded by the NIFS-collaboration research program (Grants No. NIFS20KLPF078 and No. NIFS21KLPF083). F.K. thanks Prof. S. Fritzsche of Helmholtz Institute, Jena, for his help in using RATIP. 
[1] J. D. Gillaspy, Atomic Xenon data, in EUV Sources for Lithography, edited by V. Bakshi (SPIE Press, Bellingham, Washington, 2006), Vol. 149, p. 47.

[2] P. A. C. Takman, H. Stollberg, G. A. Johansson, A. Holmberg, M. Lindblom, and H. M. Hertz, High-resolution compact X-ray microscopy, J. Microsc. 226, 175 (2007).

[3] C. Suzuki, F. Koike, I. Murakami, N. Tamura, S. Sudo, H. A. Sakaue, N. Nakamura, S. Morita, M. Goto, D. Kato, T. Nakano, T. Higashiguchi, C. S. Harte, and G. O'Sullivan, EUV spectroscopy of highly charged high $z$ ions in the large helical device plasmas, Phys. Scr. 89, 114009 (2014).

[4] C. Suzuki, I. Murakami, F. Koike, N. Tamura, H. A. Sakaue, S. Morita, M. Goto, D. Kato, H. Ohashi, T. Higashiguchi, S. Sudo, and G. O'Sullivan, Extreme ultraviolet spectroscopy and atomic models of highly charged heavy ions in the large helical device, Plasma Phys. Control. Fusion 59, 014009 (2017).

[5] S. S. Churilov, R. R. Kildiyarova, A. N. Ryabtsev, and S. V. Sadovsky, EUV spectra of Gd and Tb ions excited in laserproduced and vacuum spark plasmas, Phys. Scr. 80, 045303 (2009).

[6] D. Kilbane and G. O’Sullivan, Extreme ultraviolet emission spectra of Gd and Tb ions, J. Appl. Phys. 108, 104905 (2010).

[7] T. Otsuka, D. Kilbane, T. Higashiguchi, N. Yugami, T. Yatagai, W. Jiang, A. Endo, P. Dunne, and G. O'Sullivan, Systematic investigation of self-absorption and conversion efficiency of $6.7 \mathrm{~nm}$ extreme ultraviolet sources, Appl. Phys. Lett. 97, 231503 (2010).

[8] B. Li, T. Otsuka, T. Higashiguchi, N. Yugami, W. Jiang, A. Endo, P. Dunne, and G. O'Sullivan, Investigation of Gd and Tb plasmas for beyond extreme ultraviolet lithography based on multilayer mirror performance, Appl. Phys. Lett. 101, 013112 (2012).

[9] G. O'Sullivan and P. K. Carroll, $4 d-4 f$ emission resonances in laser-produced plasmas, J. Opt. Soc. Am. 71, 227 (1981).

[10] P. Mandelbaum, M. Finkenthal, J. L. Schwob, and M. Klapisch, Interpretation of the quasicontinuum band emitted by highly ionized rare-earth elements in the 70-100- $\AA$ range, Phys. Rev. A 35, 5051 (1987).

[11] M. Finkenthal, S. Lippmann, L. K. Huang, H. W. Moos, Y. T. Lee, N. Spector, A. Zigler, and E. Yarkoni, $\Delta n=0 n$-shell emission of rare earth ions ( $z=59$ to 70$)$ emitted from low and high density tokamak and laser produced plasmas, Phys. Scr. 41, 445 (1990).

[12] A. Sasaki, K. Nishihara, A. Sunahara, H. Furukawa, T. Nishikawa, and F. Koike, Theoretical investigation of the spectrum and conversion efficiency of short wavelength extremeultraviolet light sources based on terbium plasmas, Appl. Phys. Lett. 97, 231501 (2010).

[13] F. Koike, S. Fritzsche, and K. Nishihara, MCDF calculations for EUV-emissions of $4 d$-open shell ions based on the features of non-local exchange integrals, J. Phys.: Conf. Ser. 58, 157 (2007).

[14] G. D. O'Sullivan and R. Faulkner, Tunable narrowband soft X-ray source for projection lithography, Opt. Eng. 33, 3978 (1994).

[15] V. A. Zilitis, Theoretical study of energy level structure of ions of the isoelectronic series of gallium, Opt. Spectrosc. 81, 483 (1996) [Translated from Optika i Spektroskopia 81, 533 (1996)].
[16] D. Kato, N. Nakamura, S. Ohtani, S. Ohtani, and A. Sasaki, $Z$-dependence of photo-emission spectra for highly charged neonlike ions, Phys. Scr. T92, 126 (2001).

[17] N. Nakamura, D. Kato, and S. Ohtani, Evidence for strong configuration mixing in $n=3$ excited levels in neonlike ions, Phys. Rev. A 61, 052510 (2000).

[18] P. K. Carroll and G. O'Sullivan, Ground-state configurations of ionic species I through XVI for $z=57-74$ and the interpretation of $4 d-4 f$ emission resonances in laser-produced plasmas, Phys. Rev. A 25, 275 (1982).

[19] G. M. Zeng, H. Daido, T. Nishikawa, H. Takabe, S. Nakayama, H. Aritome, K. Murai, Y. Kato, M. Nakatsuka, and S. Nakai, Soft X-ray spectra of highly ionized elements with atomic numbers ranging from 57 to 82 produced by compact lasers, J. Appl. Phys. 75, 1923 (1994).

[20] M. Finkenthal, H. W. Moos, A. Bar-Shalom, N. Spector, A. Zigler, and E. Yarkoni, Electron-density dependence of line intensities of $\mathrm{Cu}$ I-like $\mathrm{Sm}^{33+}$ to $\mathrm{Yb}^{41+}$ emitted from tokamak and laser-produced plasmas, Phys. Rev. A 38, 288 (1988).

[21] K. B. Fournier, W. H. Goldstein, A. Osterheld, M. Finkenthal, S. Lippmann, L. K. Huang, H. W. Moos, and N. Spector, Soft $\mathrm{x}$-ray emission of galliumlike rare-earth atoms produced by high-temperature low-density tokamak and high-density laser plasmas, Phys. Rev. A 50, 2248 (1994).

[22] M. Finkenthal, A. S. Lippmann, L. K. Huang, T. L. Yu, B. C. Stratton, H. W. Moos, M. Klapisch, P. Mandelbaum, A. B. Shalom, W. L. Hodge, P. E. Phillips, T. R. Price, J. C. Porter, B. Richards, and W. L. Rowan, The spectrum of highly ionized praseodymium and dysprosium from the Texas tokamak plasma in the 50-250- $\AA$ range, J. Appl. Phys. 59, 3644 (1986).

[23] C. Suzuki, T. Kato, K. Sato, N. Tamura, D. Kato, S. Sudo, N. Yamamoto, H. Tanuma, H. Ohashi, S. Suda, G. O'Sullivan, and A. Sasaki, EUV spectra from highly charged tin ions observed in low density plasmas in LHD, J. Phys.: Conf. Ser. 163, 012019 (2009).

[24] C. Suzuki, T. Kato, H. A. Sakaue, D. Kato, K. Sato, N. Tamura, S. Sudo, N. Yamamoto, H. Tanuma, H. Ohashi, R. D’Arcy, and G. O'Sullivan, Analysis of EUV spectra of Sn XIX-XXII observed in low-density plasmas in the large helical device, J. Phys. B 43, 074027 (2010).

[25] C. Suzuki, C. S. Harte, D. Kilbane, T. Kato, H. A. Sakaue, I. Murakami, D. Kato, K. Sato, N. Tamura, S. Sudo, M. Goto, R. D'Arcy, E. Sokell, and G. O'Sullivan, Interpretation of spectral emission in the $20 \mathrm{~nm}$ region from tungsten ions observed in fusion device plasmas, J. Phys. B 44, 175004 (2011).

[26] C. Suzuki, F. Koike, I. Murakami, N. Tamura, and S. Sudo, Observation of EUV spectra from gadolinium and neodymium ions in the large helical device, J. Phys. B 45, 135002 (2012).

[27] C. Suzuki, F. Koike, I. Murakami, N. Tamura, and S. Sudo, Temperature dependent EUV spectra of Gd, Tb and Dy ions observed in the large helical device, J. Phys. B 48, 144012 (2015).

[28] C. Suzuki, F. Koike, I. Murakami, N. Tamura, and S. Sudo, Systematic observation of EUV spectra from highly charged lanthanide ions in the large helical device, Atoms 6, 24 (2018).

[29] C. Suzuki, F. Koike, I. Murakami, N. Tamura, S. Sudo, and G. O'Sullivan, Soft x-ray spectroscopy of rare-earth elements in LHD plasmas, Atoms 7, 66 (2019). 
[30] C. Suzuki, F. Koike, I. Murakami, T. Oishi, and N. Tamura, Spectra of Ga-like to $\mathrm{Cu}$-like praseodymium and neodymium ions observed in the large helical device, Atoms 9, 46 (2021).

[31] D. Kilbane, G. O'Sullivan, J. D. Gillaspy, Y. Ralchenko, and J. Reader, EUV spectra of Rb-like to $\mathrm{Cu}$-like gadolinium ions in an electron-beam ion trap, Phys. Rev. A 86, 042503 (2012).

[32] H. Ohashi, H. A. Sakaue, and N. Nakamura, Extreme ultraviolet emission spectroscopy of highly charged gadolinium ions with an electron beam ion trap, Phys. Scr. T156, 014013 (2013).

[33] D. Kilbane, G. O’Sullivan, Y. A. Podpaly, J. D. Gillaspy, J. Reader, and Y. Ralchenko, EUV spectra of Rb-like to Ni-like dysprosium ions in an electron beam ion trap, Eur. Phys. J. D 68, 222 (2014).

[34] Y. A. Podpaly, J. D. Gillaspy, J. Reader, and Y. Ralchenko, Measurements and identifications of extreme ultraviolet spectra of highly-charged Sm and Er, J. Phys. B 48, 025002 (2015).

[35] Dipti, R. Silwal, J. M. Dreiling, S. C. Sanders, E. Takács, and Y. Ralchenko, Spectroscopic analysis of $N$-intrashell transitions in Rb-like to Ni-like Yb ions, J. Phys. B 53, 145002 (2020).

[36] C. Suzuki, Dipti, Y. Yang, A. Gall, R. Silwal, S. Sanders, A. Naing, J. Tan, E. Takacs, and Y. Ralchenko, Identifications of extreme ultraviolet spectra of Br-like to ni-like neodymium ions using an electron beam ion trap, J. Phys. B 54, 015001 (2020).

[37] T. Kato, H. Funaba, K. Sato, D. Kato, M.-Y. Song, N. Yamamoto, H. Tanuma, H. Ohashi, A. Sasaki, F. Koike, K. Nishihara, K. Fahy, and G. O'Sullivan, EUV spectroscopy of $\mathrm{Xe}$ ions from the large helical device at the national institute for fusion science for stable plasmas and plasmas undergoing radiation collapse, J. Phys. B 41, 035703 (2008).

[38] C. S. Harte, C. Suzuki, T. Kato, H. A. Sakaue, D. Kato, K. Sato, N. Tamura, S. Sudo, R. D’Arcy, E. Sokell, J. White, and G. O'Sullivan, Tungsten spectra recorded at the LHD and comparison with calculations, J. Phys. B 43, 205004 (2010).

[39] Y. Takeiri et al., Extension of the operational regime of the LHD towards a deuterium experiment, Nucl. Fusion 57, 102023 (2017).

[40] Y. Takeiri, Advanced helical plasma research towards a steadystate fusion reactor by deuterium experiments in large helical device, Atoms 6, 69 (2018).

[41] S. Sudo and N. Tamura, Tracer-encapsulated solid pellet injection system, Rev. Sci. Instrum. 83, 023503 (2012).

[42] N. Tamura, S. Sudo, C. Suzuki, and H. Funaba, Creation of impurity source inside plasmas with various types of tracerencapsulated solid pellet, Plasma and Fusion Research 10, 1402056 (2015).

[43] K. Narihara, I. Yamada, H. Hayashi, and K. Yamauchi, Design and performance of the Thomson scattering diagnostic on LHD, Rev. Sci. Instrum. 72, 1122 (2001).

[44] I. Yamada, K. Narihara, H. Funaba, H. Hayashi, T. Kohmoto, H. Takahashi, T. Shimozuma, S. Kubo, Y. Yoshimura, H. Igami, and N. Tamura, Improvements of data quality of the LHD Thomson scattering diagnostics in high-temperature plasma experiments, Rev. Sci. Instrum. 81, 10D522 (2010).
[45] J. L. Schwob, A. W. Wouters, S. Suckewer, and M. Finkenthal, High-resolution duo-multichannel soft x-ray spectrometer for tokamak plasma diagnostics, Rev. Sci. Instrum. 58, 1601 (1987).

[46] N. Nakamura, J. Asada, F. J. Currell, T. Fukami, K. Motohashi, T. Nagata, E. Nojikawa, S. Ohtani, K. Okazaki, M. Sakurai, H. Shiraishi, S. Tsurubuchi, and H. Watanabe, An overview of the Tokyo electron beam ion trap, Phys. Scr. T73, 362 (1997).

[47] H. Ohashi, J. Yatsurugi, H. A. Sakaue, and N. Nakamura, High resolution extreme ultraviolet spectrometer for an electron beam ion trap, Rev. Sci. Instrum. 82, 083103 (2011).

[48] H. Ohashi, T. Higashiguchi, B. Li, Y. Suzuki, M. Kawasaki, T. Kanehara, Y. Aida, S. Torii, T. Makimura, W. Jiang, P. Dunne, G. O'Sullivan, and N. Nakamura, Tuning extreme ultraviolet emission for optimum coupling with multilayer mirrors for future lithography through control of ionic charge states, J. Appl. Phys. 115, 033302 (2014).

[49] C. Yamada, K. Nagata, N. Nakamura, S. Ohtani, S. Takahashi, T. Tobiyama, M. Tona, M. Sakurai, A. P. Kavanagh, and F. J. Currell, Injection of metallic elements into an electron beam ion trap using a Knudsen cell, Rev. Sci. Instrum. 77, 066110 (2006).

[50] C. M. Brown, J. F. Seely, D. R. Kania, B. A. Hammel, C. A. Back, R. W. Lee, A. Barshalom, and W. E. Behring, Wavelengths and energy levels for the $\mathrm{Zn}$ I isoelectronic sequence Sn20+ through U62+, At. Data Nucl. Data Tables 58, 203 (1994).

[51] A. Kramida, Yu. Ralchenko, J. Reader, and NIST ASD Team, NIST Atomic Spectra Database, Version 5.8, National Institute of Standards and Technology, Gaithersburg, MD, 2020, https: //physics.nist.gov/asd.

[52] K. P. Dere, E. Landi, H. E. Mason, B. C. M. Fossi, and P. R. Young, CHIANTI-an atomic database for emission lines. I. Wavelengths greater than 50 A, Astron. Astrophys. Suppl. Ser. 125, 149 (1997).

[53] E. Landi, G. D. Zanna, P. R. Young, K. P. Dere, and H. E. Mason, CHIANTI-an atomic database for emission lines. XII. version 7 of the database, Astrophys. J. 744, 99 (2012).

[54] F. Parpia, C. Fischer, and I. Grant, Grasp92: A package for large-scale relativistic atomic structure calculations, Comput. Phys. Commun. 94, 249 (1996).

[55] S. Fritzsche, The Ratip program for relativistic calculations of atomic transition, ionization and recombination properties, Comput. Phys. Commun. 183, 1525 (2012).

[56] K. Dyall, I. Grant, C. Johnson, F. Parpia, and E. Plummer, Grasp: A general-purpose relativistic atomic structure program, Comput. Phys. Commun. 55, 425 (1989).

[57] P. Jönsson, X. He, C. Froese Fischer, and I. Grant, The Grasp2k relativistic atomic structure package, Comput. Phys. Commun. 177, 597 (2007).

[58] C. Froese Fischer, G. Gaigalas, P. Jönsson, and J. Bieroń, GRASP2018-A Fortran 95 version of the general relativistic atomic structure package, Comput. Phys. Commun. 237, 184 (2019).

[59] C. F. Fischer, T. Brage, and P. Jonsson, Atomic Structure: An MCHF Approach (Institute of Physics, University of Reading, Berkshire, 1997). 
[60] F. El-Sayed, Energy levels and transition rates for Ga-like ions (Xe XXIV-Pr XXIX), J. Appl. Spectrosc. 82, 487 (2015).

[61] F. El-Sayed and S. M. Attia, Energies, wavelengths, and transition rates for Ga-like ions (Nd XXX-Tb XXXV), J. Appl. Spectrosc. 83, 126 (2016).

[62] F. Hu, J. Yang, C. Wang, L. Jing, S. Chen, G. Jiang, H. Liu, and L. Hao, Multiconfiguration Dirac-Fock calculations on multi-valence-electron systems: Benchmarks on Ga-like ions, Phys. Rev. A 84, 042506 (2011).

[63] Y. Hahn, in Theory of Dielectronic Recombination (Academic Press, New York, 1985), pp. 123-196.

[64] Y. Hahn and K. Lagattuta, Dielectronic recombination and related resonance processes, Phys. Rep. 166, 195 (1988). 